Received: 10.04 .2020

Revised: 15.05 .2020

Accepted: 19.06 .2020

DOI: $10.17804 / 2410-9908.2020 .3 .047-060$

\title{
ON MODELS FOR ESTIMATING THE POST-RADIATION DEFECT STATE OF THE $\gamma^{\prime}$-PHASE
}

\author{
V. I. Bobrovskii \\ M.N. Miheev Institute of Metal Physics, Ural Branch of the Russian Academy of Sciences, \\ 18 S. Kovalevskoy St., Ekaterinburg, 620990, Russian Federation \\ (iD https://orcid.org/0000-0002-4692-8889 bobrovskii@imp.uran.ru \\ *Corresponding author. E-mail: bobrovskii@imp.uran.ru \\ Address for correspondence: 18, S. ul. Kovalevskoy, Ekaterinburg, 620990, Russian Federation \\ Tel.: +7 (343) 3740003
}

Processes that take place in the precipitates of $\gamma^{\prime}$-phase under irradiation with fast neutrons are topical and draw attention when one searches for ways of improving radiation resistance of structural reactor steels. A special feature of these processes is that the formation of vacancies and interstices proceeds at the background of a disordering of the initial crystal lattice, which manifests itself in the formation of antisite defects. X-ray and neutron diffraction techniques are efficient tools of studying changes that occur in the post-radiation structural state of such systems. However, interpretation of the experimental results calls for more complicated models for the description of structural effects exerted by accumulation of radiation defects in the material than those developed before for elementary metals. Several models are proposed in this paper.

Keywords: neutron irradiation, radiation-induced processes, vacancies, interstitials, antisite defects.

\section{Acknowledgment}

The research was carried out at the IMP Neutron Material Science Complex within the state assignment of the Ministry of Science and Higher Education of the Russian Federation (theme "Neutron" No. AAAA-A19-119112590082-1).

\section{References}

1. Alab'yev V.M., Vologin V.G., Dubinin S.F., Lapin S.S., Parkhomenko V.D., Sagaradze V.V. Neutron diffraction and electron microscopic investigation of decomposition and radiationinduced ageing of Cr-Ni-Ti austenitic alloys. Physics of Metals and Metallography, 1990, vol. 70, no. 2, pp. 131-137.

2. $\quad$ Sagaradze V.V., Nalesnik V.M., Lapin S.S., Aliabev V.M. Precipitation hardening and radiation damageability of austenitic stainless steels. Journal of Nuclear Materials, 1993, vol. 202, no. 1-2, pp. 137-144. DOI: 10.1016/0022-3115(93)90036-X.

3. Okita T., Wolfer W.G., Garner F.A., Sekimura N. Effects of titanium additions to austenitic ternary alloys on microstructural evolution and void swelling. Philosophical Magazine, 2005, vol. 85, no. 18, pp. 2033-2048. DOI: 10.1080/14786430412331331871.

4. Voronin V.I., Berger I.F., Goshchitskii B.N. Structural changes in a model alloy after irradiation of Fe62Ni35Ti3 with fast neutrons and isochronous temperature annealing. Physics of Metals and Metallography, 2012, vol. 113, no. 9, pp. 878-882. DOI: 10.1134/S0031918X12090141.

5. Mosbrucker P.L., Brown D.W., Anderoglu O., Balogh L., Maloy S.A., Sisneros T.A., Dippel A.C. Neutron and X-ray diffraction analysis of the effect of irradiation dose and temperature on 
microstructure of irradiated HT-9 steel. Journal of Nuclear Materials, 2013, vol. 443, no. 1, pp. 522-530. DOI: 10.1016/j.jnucmat.2013.07.065.

6. Voronin V.I., Arbuzov V.L., Bobrovskii V.I., Danilov S.E., Kozlov K.A., Proskurnina N.V., Sagaradze V.V. Peculiarities of radiation-induced processes in the Cr-Ni-Mo austenitic steels studied by neutron diffraction. Diagnostics, Resource and Mechanics of materials and structures, 2015, iss. 5, pp. 80-89. DOI: 10.17804/2410-9908.2015.5.080-089. Available at: http://dreamjournal.org/issues/2015-5/2015-5_46.html (accessed: 17.08.2020).

7. Proskurnina N.V., Bobrovskii V.I., Goshchitskii B.N., Volkov A.Yu., Voronin V.I. Radiation induced disordering in Cu3Au. Radiation Physics and Chemistry, 2020, vol. 170, pp. 108654. DOI: 10.1016/j.radphyschem.2019.108654.

8. Cahn R.W. Lattice parameter changes on disordering intermetallics. Intermetallics, 1999, vol. 7, pp. 1089-1094. DOI: 10.1016/S0966-9795(99)00035-7.

9. Bhatia M.L., Cahn R.W. Lattice parameter and volume changes on disordering. Intermetallics, 2005, vol. 13, pp. 474-483. DOI: 10.1016/S0966-9795(99)00035-7.

10. Konobeevskii, S.T., Deistvie izluchenii na materialy. Vvedenie v radiatsionnoe materialovedenie [The Effects of Radiation on Materials. Introduction to the Radiation Material Science]. Moscow, Atomizdat Publ., 1967. (In Russian).

11. Kovács I., Elsayed H. Point defects in metals. J. Mater Sci., 1976, vol. 11, pp. 529-559. DOI: 10.1007/BF00540934.

12. Beneagoub A., Thome L. Amorphization Mechanisms in Ion-Bombarded Metallic Alloys. Phys. Rev. B: Condens. Matter., 1988, vol. 38, pp. 10205-10216. DOI: 10.1103/PhysRevB.38.10205.

13. Eshelby J.D. The Continuum Theory of Lattice Defects. Solid State Physics, 1956, vol. 3, pp. 79-144. DOI: 10.1016/S0081-1947(08)60132-0.

14. Was G.S. Fundamentals of Radiation Materials Science. Metals and Alloys, second ed., New York, Springer, 2017, 1002 p. ISBN 978-1-4939-3436-2. DOI: 10.1007/978-1-4939-3438-6. 
Подана в журнал: 10.04 .2020

УДК 669.15-194.56:620.183.6:539.2:620.181

DOI: $10.17804 / 2410-9908.2020 .3 .047-060$

\title{
К МОДЕЛЯМ ДЛЯ ОЦЕНКИ ПОСТРАДИАЦИОННОГО ДЕФЕКТНОГО СОСТОЯНИЯ $\gamma^{\prime}$-ФАЗЫ
}

\author{
В. И. Бобровский \\ ФГБУН Институт физики металлов имени М.Н. Михеева Уральского отделения Российской академии наук, \\ 18, ул. С. Ковалевской, г. Екатеринбург, Российская Федераиия \\ (DD https://orcid.org/0000-0002-4692-8889 @ bobrovskii@imp.uran.ru \\ *Ответственный автор. Электронная почта: bobrovskii @imp.uran.ru \\ Адрес для переписки: ул. С. Ковалевской, 18, 620990, г. Екатеринбург, Российская Федерация \\ Тел.: +7 (343) 374-00-03; факс: 374-00-03
}

Процессы, возникающие в преципитатах $\gamma^{\prime}$-фазы при облучении быстрыми нейтронами представляют значительный интерес с точки зрения поиска путей улучшения радиационной стойкости конструкционных реакторных сталей. Особенностью этих процессов является то, что образование вакансий и междоузлий протекает в них на фоне разупорядочения исходной решетки, реализующегося в виде формирования антиструктурных дефектов. Рентгеновские и нейтронные дифракционные методы являются эффективным инструментом изучения изменений пострадиационного структурного состояния таких систем. Однако интерпретация экспериментальных результатов требует более сложных моделей для описания структурных эффектов от накопления радиационных дефектов в материале, нежели развитые ранее для простых металлов. Несколько вариантов таких моделей предложены в работе.

Ключевые слова: нейтронное облучение, радиационно-индуцированные процессы, вакансии, междоузлия антиструктурные дефекты.

\section{1. Введение}

Каскадообразующее облучение быстрыми нейтронами способно создавать в материалах неравновесные дефектные состояния зачастую недостижимые другими методами. Особенно ярко это проявляется в многокомпонентных системах, где под действием облучения могут изменяться фазовый состав и дислокационная структура, происходить радиационноиндуцированная сегрегация атомов, формироваться или наоборот растворяться различные преципитаты, а в дополнение к этому возникать заметные концентрации точечных дефектов. К таким материалам, например, относятся аустенитные конструкционные реакторные стали, использующиеся для изготовления оболочек топливных элементов реакторов на быстрых нейтронах. Основным недостатком этих сталей является вакансионное распухание, развивающееся в них при высоких дозах нейтронного облучения. В качестве одного из средств борьбы с этим явлением предлагают преципитаты $\gamma^{\prime}$-фазы $\mathrm{Ni}_{3} \mathrm{Ti}$, призванные служить стоками точечных дефектов и создаваемые в материале путем предварительного старения [1-6]. Однако следует отметить, что процессы формирования дефектных структур и, в частности, радиационно-индуцированные явления в этих образованиях изучены совершенно недостаточно и требуют отдельных углубленных исследований. Полученные экспериментальные результаты по воздействию облучения быстрыми нейтронами на модельное, изоструктурное $\gamma^{\prime}$-фазе соединение $\mathrm{Cu}_{3} \mathrm{Au}$ [7], показали весьма интересное радиационное поведение его структурных характеристик, причем значительно отличающееся от наблюдавшегося ранее в разупорядоченном термическим способом соединении $\mathrm{Ni}_{3} \mathrm{Al}[8,9]$. 
Особенностью интерметаллидов $\mathrm{Ni}_{3} \mathrm{Al}, \mathrm{Ni}_{3} \mathrm{Ti}$ и изоструктурного им высокоупорядоченного сплава $\mathrm{Cu}_{3} \mathrm{Au}$ является то, что образование вакансий и междоузлий протекает на фоне разупорядочения исходной решетки, реализующегося в виде формирования антиструктурных дефектов, т. е. обмена позициями между атомами сортов А и В в системе $\mathrm{A}_{3} \mathrm{~B}$. Наиболее удобными инструментами исследования этих эффектов являются нейтронно- и рентгеноструктурный анализы, позволяющие фиксировать изменения как дальнего порядка в системе, так и параметров решетки.

Как показал наш опыт исследований, для интерпретации полученных результатов необходимы хотя бы полукачественые модели, подобные уже развитым для простых металлов $[10,11]$. Цель работы - построение таких моделей, пригодных для описания многокомпонентных систем и одновременного протекания нескольких явлений.

\section{2. Постановка задачи, результаты и обсуждение}

Рассмотрим для начала систему, содержащую только антиструктурные дефекты. В состоянии полного упорядочения атомы сорта А занимают узлы типа (А), а атомы сорта В-узлы типа (B), образуя решетку типа $L 1_{2}$, которая при полном разупорядочении по позициям превращается в простую ГЦК-структуру (рис. 1).

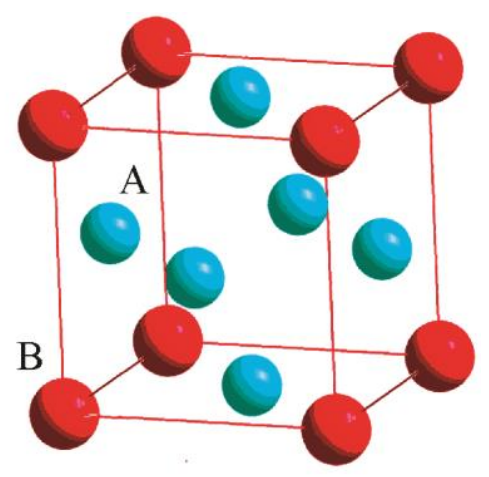

Рис. 1. Структура решетки $\gamma^{\prime}$-фазы

Для характеризации степени разупорядочения подобных бинарных систем обычно используется параметр порядка по Брэггу-Вильямсу, определяемый как:

$$
S=\frac{p_{A}^{(A)}-C_{A}}{1-C_{A}}=\frac{p_{B}^{(B)}-C_{B}}{1-C_{B}},
$$

где $C_{A}$ и $C_{B}$ - концентрации атомов сортов А и В в системе; $p_{A}^{(A)}-$ доля узлов сорта $(\mathrm{A})$, фактически занятых атомами сорта $\mathrm{A} ; p_{B}^{(B)}$ - доля узлов сорта $(\mathrm{B})$, фактически занятых атомами сорта В.

То есть $p_{A}^{(A)}$ и $p_{B}^{(B)}-$ это просто числа заполнения позиций $(\mathrm{A})$ и $(\mathrm{B})$ атомами сортов А и В, или, что то же самое, вероятности обнаружить в позиции типа (А) атом сорта А, а в позиции типа (В) - атом сорта В. Соответственно $p_{A}^{(B)}$ и $p_{B}^{(A)}$ - это вероятности обнаружить в позиции типа (B) атом сорта А, и наоборот.

В состоянии полного упорядочения $p_{A}^{(A)}=p_{B}^{(B)}=1$, соответственно и $S=1$. При полном разупорядочении $p_{A}^{(A)}=C_{A} ; p_{B}^{(B)}=C_{B}$ и $S=0$. 
С учетом очевидных соотношений:

$$
\left\{\begin{array}{c}
C_{A}+C_{B}=1 \\
p_{A}^{(A)}+p_{B}^{(A)}=1 \\
p_{A}^{(B)}+p_{B}^{(B)}=1
\end{array}\right.
$$

Легко получить:

$$
S=1-\frac{p_{A}^{(B)}}{C_{A}}=1-\frac{p_{B}^{(A)}}{C_{B}}
$$

что для системы $\mathrm{A}_{3} \mathrm{~B}$ имеет вид:

$$
S=1-\frac{4}{3} p_{A}^{(B)}=1-4 p_{A}^{(B)} .
$$

Далее рассмотрим несколько различных моделей радиационного разупорядочения данного материала.

Идеализированная гетерогенная модель. Предположим, что вследствие каскадообразующего характера нейтронного облучения в результате каждого отдельного каскада возникает область с полным разупорядочением по антиузельным дефектам. При этом повторное полное или частичное перекрытие таких каскадов на такое разупорядочение уже не влияет. Считаем, что из $N$ элементарных ячеек кристаллита с исходной совершенной структурой в разупорядоченных областях оказывается $N_{d}$ ячеек. Остальная часть кристаллита остается полностью упорядоченной. Обозначим относительную концентрацию разупорядоченных ячеек в системе:

$$
C_{d}=\frac{N_{d}}{N}
$$

Нетрудно понять, что средняя вероятность обнаружить атом сорта В в позиции (А) для такой системы равна:

$$
p_{B}^{(A)}=C_{d} C_{B}+\left(1-C_{d}\right) \cdot 0
$$

Отсюда:

$$
S=1-C_{d}
$$

Будем обозначать параметр решетки, содержащей только антиструктурные дефекты, через $a_{0}(S)$. В случае полностью упорядоченной решетки он будет выглядеть как $a_{0}(1)$, а для полностью разупорядоченной - как $a_{0}(0)$. Нижний индекс введен здесь, чтобы подчеркнуть отсутствие иных дефектов, помимо антиструктурных, и понадобится нам впоследствии. Тогда для объема всего кристалла очевидно выполняется:

$$
N a_{0}^{3}(S)=C_{d} N a_{0}^{3}(0)+\left(1-C_{d}\right) N a_{0}^{3}(1),
$$

где через $a_{0}(S)$ мы обозначили среднее по объему кристаллита значение параметра решетки. 
Обозначив $\Delta a_{0}(S)=a_{0}(S)-a_{0}(1)$, легко получить, что с точностью до первого порядка выполняется:

$$
\frac{\Delta a_{0}(S)}{a_{0}(1)}=C_{d} \frac{a_{0}(0)-a_{0}(1)}{a_{0}(1)}=(1-S) \frac{a_{0}(0)-a_{0}(1)}{a_{0}(1)} .
$$

То есть в рамках такой гетерогенной модели относительное изменение параметра решетки от параметра порядка носит линейный характер. При этом аналогичная зависимость от флюенса нейтронов $\Phi$, вызвавших такое разупорядочение, нелинейна вследствие перекрытия каскадов и может быть аппроксимирована [12]:

$$
N_{d}=N\left(1-\exp \left(-{ }_{6} \Phi\right)\right)
$$

где коэффициент $\beta$ определяется величиной сечений рассеяния ядер атомов, входящих в состав материала.

При других методах разупорядочения, например термическом, накопление антиструктурных дефектов может идти гомогенно по объему кристалла. Но и в этом случае, если обратиться к формуле (3) и предположить, что параметр ячейки меняется в первом приближении линейно с изменением вероятности $p_{A}^{(B)}$ и, соответственно, ионного радиуса атома в позиции (В) [8], то:

$$
p_{A}^{(B)}=C_{A}(1-S)=l_{A}^{(B)}\left(a_{0}(S)-a_{0}(1)\right) .
$$

Значению $S=0$ будет соответствовать:

$$
l_{A}^{(B)}\left(a_{0}(0)-a_{0}(1)\right)=C_{A} .
$$

Так что из (11) и (12) вновь получаем:

$$
\frac{a_{0}(S)-a_{0}(1)}{a_{0}(1)}=(1-S) \frac{a_{0}(0)-a_{0}(1)}{a_{0}(1)} .
$$

Предположим, что в дополнение к антиузельным дефектам в кристалле образуются некоторые концентрации по типу дефектов Шоттки и пар Френкеля, вносящие свой аддитивный вклад в величину параметра решетки $a=a\left(S ; C_{V} ; C_{I}\right) \equiv a_{C}(S)$. Здесь $C_{V}$ и $C_{I}-$ концентрации вакансий и междоузлий в кристалле, обусловленные наличием вышеупомянутых дефектов. Присутствие дополнительных точечных дефектов мы для уменьшения громоздкости формул будем указывать индексом «С», т. е. в системе обозначений:

$$
\left\{\begin{array}{c}
a(S ; 0 ; 0)=a_{0}(S) \\
a\left(0 ; C_{V} ; C_{I}\right)=a_{c}(0) \\
a(0 ; 0 ; 0)=a_{0}(0)
\end{array}\right.
$$

Теперь оценим влияние дефектов Шоттки и Френкеля на параметр $a$.

Распределение точечных дефектов по кристаллиту рассмотрим сначала в рамках гомогенной модели, полагая, что независимо от характера распределения антиструктурных дефектов (гомогенного или гетерогенного) оно однородно по объему кристаллита, а концентрации этих дефектов малы.

Среднее изменение объема кристаллита за счет релаксации решетки $[13,14]$ при появлении в ней единственной вакансии считаем равным: 


$$
\Delta v_{V}=r_{V} a_{0}^{3}(S) / 4
$$

При этом подразумевается, что при получении коэффициента $r_{V}(S)$ проведено усреднение по вероятностям образования вакансии в позициях (А) и (B), т. е. $3 / 4$ и 1/4. Аналогично изменение объема образца при появлении единственного междоузлия:

$$
\Delta v_{I}=r_{I} a_{0}^{3}(S) / 4
$$

В формулах (15)-(16) величина релаксации решетки выражена в единицах объема доли элементарной ячейки, приходящейся на одну кристаллографическую позицию, т. е. среднего атомного объема системы. Строго говоря, при теоретическом расчете значений величин $r_{V}(S)$ и $r_{I}(S)$, чтобы полностью учесть $S$, усреднения следует проводить по соответствующему ансамблю конфигураций ближнего окружения дефекта. Однако зависимости величин $r_{V}(S)$ и $r_{I}(S)$ от параметра $S$, как представляется, являются эффектами высшего порядка малости по сравнению с рассматриваемыми здесь и поэтому будут в дальнейшем опущены.

Для вакансий, входящих в дефекты Шоттки, их появление сопровождается выходом междоузлий на поверхность кристаллита, где они формируют некую кристаллическую структуру. Поскольку экспериментально при разупорядочении рассматриваемых систем появление новых фаз не зафиксировано, считаем, что формируемая ими структура остается без изменений, но ввиду стохастичности выхода междоузлий оказывается в полностью разупорядоченном состоянии, т. е. отвечает $S=0$. Для формирования такой ячейки с объемом $a_{C}^{3}(0)$ требуются 4 междоузлия (считаем, что концентрации точечных дефектов в такой «корочке» те же самые, что и по всему кристаллиту). Тогда изменение объема образца за счет $n_{S}$ дефектов Шоттки составит:

$$
\Delta V_{S}=\left(n_{S} r_{V} a_{0}^{3}(S)+n_{S} a_{C}^{3}(0)\right) / 4
$$

А для $n_{F}$ дефектов Френкеля, состоящих из пары вакансия-междоузлие:

$$
\Delta V_{F}=n_{F}\left(r_{V}+r_{I}\right) a_{0}^{3}(S) / 4
$$

учитывая, что число пар Френкеля равно числу междоузлий, т. е. $n_{F}=n_{I}$, а полное число вакансий $n_{V}=n_{S}+n_{F}$, получаем для полного изменения объема решетки за счет дефектов:

$$
\Delta V=\left(n_{V} r_{V}+n_{I} r_{I}\right) a_{0}^{3}(S) / 4+\left(n_{V}-n_{I}\right) a_{C}^{3}(0) / 4 \text {. }
$$

Удобно ввести коэффициент $\varepsilon(S)$, описывающий относительное изменение объема решетки, оставшееся до ее полного разупорядочения и наполнения дополнительными дефектами:

$$
e(S)=\frac{a_{C}^{3}(0)-a_{0}^{3}(S)}{a_{0}^{3}(S)}=3 \frac{a_{C}(0)-a_{0}(S)}{a_{0}(S)}=3 \frac{a\left(0 ; C_{V} ; C_{I}\right)-a(S ; 0 ; 0)}{a(S ; 0 ; 0))} .
$$

Отметим, что этот коэффициент отражает особенности принятой модели разупорядочения бинарной системы. При аналогичном рассмотрении эффектов в простых металлах он вообще не возникает. Сопоставление его вклада, который сразу видится малым, с другими составляющими будет выполнено позднее, а пока мы сохраняем его как специфическую особенность многокомпонентных систем.

Тогда, если обозначить концентрации вакансий и междоузлий $C_{V}=n_{V} / 4 N ; C_{I}=n_{I} / 4 N$, получаем: 


$$
\Delta V=N a_{0}^{3}(S)\left[\left(C_{V}-C_{I}\right)(1+e)+\left(C_{V} r_{V}+C_{I} r_{I}\right)\right]
$$

Откуда следует, что относительное изменение объема кристаллита за счет появления точечных дефектов:

$$
\frac{\Delta V}{V_{0}}=\frac{\Delta V}{N a_{0}^{3}(S)}=\left[\left(C_{V}-C_{I}\right)(1+e)+\left(C_{V} r_{V}+C_{I} r_{I}\right)\right] .
$$

Рассматривая кубический кристаллит с ребром $L\left(V=L^{3}\right)$, с точностью до первого порядка малости по $\Delta L$ получим для относительного удлинения кристаллита за счет вакансий и междоузлий:

$$
\frac{\Delta L}{L_{0}}=\frac{1}{3} \frac{\Delta V}{V_{0}}=\frac{1}{3}\left[\left(C_{V}-C_{I}\right)(1+e)+\left(C_{V} r_{V}+C_{I} r_{I}\right)\right]
$$

Теперь учтем, что полный объем кристаллита, содержащего $N_{1}=N+\frac{\left(n_{V}-n_{I}\right)}{4}=$ $=N\left[1+\left(C_{V}-C_{I}\right)\right]$ ячеек, равен:

$$
V_{1}=N a_{0}^{3}(S)+\Delta V=N a_{0}^{3}(S)\left[1+\left(C_{V}-C_{I}\right)(1+e)+\left(C_{V} r_{V}+C_{I} r_{I}\right)\right]
$$

Тогда объем $v_{c}=a_{C}^{3}(S)$, приходящийся на одну элементарную ячейку кристаллита с точностью до первого порядка по концентрациям равен:

$$
v_{c}=\frac{V_{1}}{N_{1}}=\frac{N a_{0}^{3}(S)+\Delta V}{N_{1}} \approx a_{0}^{3}(S)\left\{1+\left[\left(C_{V}-C_{I}\right) e+\left(C_{V} r_{V}+C_{I} r_{I}\right)\right]\right\}
$$

Откуда очевидно, что обусловленное вакансиями и междоузлиями относительное изменение параметра решетки равно:

$$
\frac{\Delta a}{a_{0}(S)}=\frac{\left.a\left(S ; C_{I} ; C_{V}\right)-a(S ; 0 ; 0)\right)}{a(S ; 0 ; 0))}=\frac{1}{3}\left[\left(C_{V}-C_{I}\right) e+\left(C_{V} r_{V}+C_{I} r_{I}\right)\right]
$$

Естественно, при этом выполняется каноническое:

$$
3\left(\frac{\Delta L}{L_{0}}-\frac{\Delta a}{a_{0}}\right)=C_{V}-C_{I}
$$

Возвращаясь к формуле (26), видим:

$$
a_{C}(S)=a_{0}(S)\left\{1+\frac{1}{3}\left[\left(C_{V}-C_{I}\right) e+\left(C_{V} r_{V}+C_{I} r_{I}\right)\right]\right\}
$$

Используя (13), получаем:

$$
a_{C}(S)=a_{0}(1)+(1-S)\left[a_{0}(0)-a_{0}(1)\right]+\frac{a_{0}(1)}{3}\left[\left(C_{V}-C_{I}\right) e+\left(C_{V} r_{V}+C_{I} r_{I}\right)\right]
$$

Откуда полное относительное приращение решетки, отсчитываемое от параметра решетки полностью упорядоченного бездефектного кристалла $a_{0}(1)=a(1 ; 0 ; 0)$, равно: 


$$
\left(\frac{\Delta a}{a_{0}(1)}\right)_{\mathrm{y}}=\left(\frac{\Delta a}{a_{0}(1)}\right)_{\mathrm{AS}}+\left(\frac{\Delta a}{a_{0}(1)}\right)_{\mathrm{VI}}
$$

Вклад, обусловленный антиструктурными дефектами, равен:

$$
\left(\frac{\Delta a}{a_{0}(1)}\right)_{\mathrm{A} S}=(1-S) \frac{a_{0}(0)-a_{0}(1)}{a_{0}(1)}=k_{A S} x,
$$

а вклад за счет вакансий и междоузлий имеет вид:

$$
\left(\frac{\Delta a}{a_{0}(1)}\right)_{V I}=\frac{1}{3}\left[\left(C_{V}-C_{I}\right) e+\left(C_{V} r_{V}+C_{I} r_{I}\right)\right] .
$$

Здесь также введены переменная $x=1-S$ и коэффициент $k_{A S}=\frac{a_{0}(0)-a_{0}(1)}{a_{0}(1)}$

Сделаем оценки величины различных вкладов. Как можно усмотреть из (31), вклад от антиструктурных дефектов описывается простой линейной зависимостью от $x$. На практике коэффициент $k_{A S}$, стоящий при этой линейной зависимости, составляет величину порядка $\sim 2 \cdot 10^{-3}$ [7]. Введенный в (20) коэффициент $e$ был призван учесть изменение параметра решетки за счет вакансий и междоузлий и полного доразупорядочения в смысле антиструктурных дефектов в «корочке» кристаллита, образованной междоузлиями из дефектов Шоттки. Ясно, что по порядку величины он совпадает с $k$ :

$$
e_{\max }=3 \frac{a_{0}(0)-a_{0}(1)}{a_{0}(1)}=3 k_{A S} .
$$

Однако, в отличие от (31), этот коэффициент стоит не при переменной $x$, изменяющейся в интервале $(0 ; 1)$, а при малых концентрациях $C_{v} ; C_{I}$. Отметим также, что своего максимального значения этот коэффициент достигает вблизи состояния кристалла с $S=1$. Следует принять во внимание, что и антиструктурные дефекты, и дефекты Шоттки, и Френкеля развиваются в системе одновременно под воздействием разупорядочивающего нейтронного облучения. Это означает, что, по крайней мере, вблизи идеально упорядоченного состояния кристалла концентрация этих дефектов должна быть пропорциональна флюенсу облучения, а значит, как очевидно из (10), величине $x$. Если сравнивать вклады первого и второго слагаемого в квадратных скобках в (32), то можно указать, что, как показывает опыт [14], для ГЦКметаллов коэффициенты, описывающие релаксацию решетки на появление вакансии и междоузлия, обычно составляют: $r_{I} \sim(1 \div 2) ; r_{V} \sim-(0,1 \div 0,2)$. Все это показывает, что в рамках точности проводившихся здесь вычислений первое слагаемое в квадратной скобке в (32) должно быть опущено.

Тогда, вводя обозначения $C_{V}=g_{V} x ; C_{I}=g_{I} x$, где коэффициенты $g_{V} ; g_{I}$ для достаточно малых значений $x$ могут считаться постоянными, получаем:

$$
\left(\frac{\Delta a}{a_{0}(1)}\right)_{V I}=\frac{1}{3}\left(g_{V} r_{V}+g_{I} r_{I}\right) x .
$$

Хорошо известно, что в состоянии термодинамического равновесия концентрация вакансий в металлах мала, а концентрацией междоузельных атомов можно просто пренебречь, т. е. $C_{V} \gg C_{I}$. Однако разупорядочение быстрыми нейтронами способно создавать в материалах необычные неравновесные состояния, недостижимые другими методами. Конечно, следует признать, что на практике, оперируя с образцами, извлеченными из облучательных ка- 
налов реактора и прошедшими период выдержки для уменьшения наведенной радиоактивности, мы наблюдаем состояния систем уже после некоторого пострадиационного отжига. При этом представляется разумным предположить, что все-таки существуют некие предельные значения для концентраций $C_{V}$ и $C_{I}$ в системе, поэтому по достижении некоторого значения $x_{0}$ зависимость (34) должна сменяться режимом насыщения, т. е. в рамках рассматриваемой модели в простейшем варианте графики для относительных приращений решетки представлены на рис. 2.

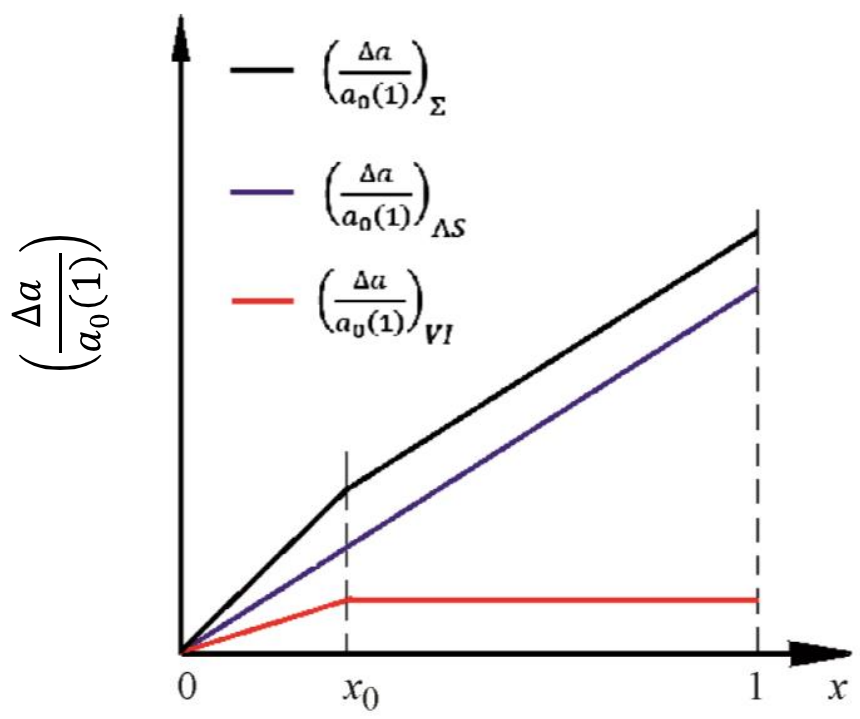

Рис. 2. Вклады в относительное приращение параметра решетки бинарной системы $\mathrm{A}_{3} \mathrm{~B}$ при разупорядочении быстрыми нейтронами в рамках принятой модели

Такая полукачественная картина хорошо согласуется с экспериментально наблюдаемыми результатами разупорядочения системы $\mathrm{Cu}_{3} \mathrm{Au}$, приведенными в [7]. Данный сплав отличается весьма высокой способностью к антиструктурному разупорядочению (легко достижимому и термическим, и механическим, и радиационным воздействиями), что свидетельствует о низких значениях энергий миграции слагающих его атомов, т. е. о их высокой способности к диффузии. Вследствие этого для данного материала модель гомогенного распределения вакансий и междоузлий по объему, по крайней мере, на начальном этапе разупорядочения, представляется вполне разумной.

В модель можно вносить и дальнейшие качественные усовершенствования. Например, учесть образование вакансионных кластеров. Предположим, что часть вакансий образуют достаточно крупные кластеры. Будем считать, что такие поры не создают внутренних напряжений в решетке, а просто образуют в ней систему пустот, и, соответственно, дополним нашу модель.

Считаем, что общее количество междоузлий и вакансий, изначально возникающих при образовании дефектов Шоттки и Френкеля, равно $n_{I}^{(i)}$ и $n_{V}^{(i)}$ соответственно. Очевидно, что $n_{I}^{(i)}=n_{V}^{(i)}$. Первые в дальнейшем разобьются на ушедшие при формировании дефектов Шоттки на поверхность кристаллита в количестве $n_{S}$ и внутренние междоузлия $n_{I}$, а вторые на одиночные вакансии $n_{V}$ и вакансии, сформировавшие поры $n_{P}$ :

$$
\left\{\begin{array}{l}
n_{I}^{(i)}=n_{S}+n_{I} \\
n_{V}^{(i)}=n_{V}+n_{P}
\end{array} .\right.
$$


Откуда следует:

$$
n_{S}=\left(n_{V}-n_{I}\right)+n_{P}
$$

Выражая происходящие при этом внешние изменения объема кристаллита аналогично (16)-(18), но пренебрегая эффектами, описывавшимися введением коэффициента $\varepsilon$, получаем:

$$
\Delta V=n_{V} r_{V} \frac{a_{0}^{3}}{4}+n_{I} r_{I} \frac{a_{0}^{3}}{4}+n_{S} \frac{a_{0}^{3}}{4}
$$

Учитывая (36), получаем:

$$
\left(\frac{\Delta V}{V_{0}}\right)_{V I}=\frac{\Delta V}{N a_{0}^{3}}=\left(C_{V}-C_{I}\right)+\left(C_{V} r_{V}+C_{I} r_{I}\right)+C_{P}
$$

и, соответственно, относительное удлинение кристаллита:

$$
\left(\frac{\Delta L}{L_{0}}\right)_{V I}=\frac{1}{3}\left[\left(C_{V}-C_{I}\right)+\left(C_{V} r_{V}+C_{I} r_{I}\right)+C_{P}\right]
$$

где $C_{P}=\frac{n_{P}}{4 N}-$ относительное число узельных позиций, вошедших в поры.

Для определения среднего параметра решетки с дефектами поделим часть объема кристаллита $\bar{V}$ за вычетом пор на количество ячеек в ней $\bar{N}$ :

$$
\begin{gathered}
\bar{V}=V_{0}+\Delta V-\frac{n_{P} a_{0}^{3}}{4}=N a_{0}^{3}\left[1+\left(C_{V}-C_{I}\right)+\left(C_{V} r_{V}+C_{I} r_{I}\right)\right] \\
\bar{N}=N+\frac{n_{S}}{4}-\frac{n_{P}}{4}=N\left[1+\left(C_{V}-C_{I}\right)\right] .
\end{gathered}
$$

Тогда с точностью до первого порядка малости по концентрациям получаем:

$$
\begin{gathered}
a^{3}=\frac{\bar{V}}{\bar{N}}=a_{0}^{3}\left(1+\left(C_{V} r_{V}+C_{I} r_{I}\right)\right) ; \\
\left(\frac{\Delta a}{a_{0}}\right)_{V I}=\frac{1}{3}\left(C_{V} r_{V}+C_{I} r_{I}\right) .
\end{gathered}
$$

Интересно, что соотношение (27) теперь принимает вид:

$$
3\left(\frac{\Delta L}{L_{0}}-\frac{\Delta a}{a_{0}}\right)_{V I}=\left(C_{V}-C_{I}\right)+C_{P} .
$$

Откуда видно, что в случае, когда поведение концентраций $C_{V}$ и $C_{I}$ соответствует графикам на рис. 2 , т. е. до достижения критического значения параметра $x_{0}$ их зависимости от $x$ линейны, а затем они достигают предельных значений и превращаются в константы, из зависимости (44) можно судить о развитии вакансионных кластеров в системе, т. е. формулы (38), (39) и (44) дают простейшую модель вакансионного распухания. То есть при наличии 
поведения параметра решетки как на рис. 2, можно интерпретировать зависимость (44) согласно рис. 3 :

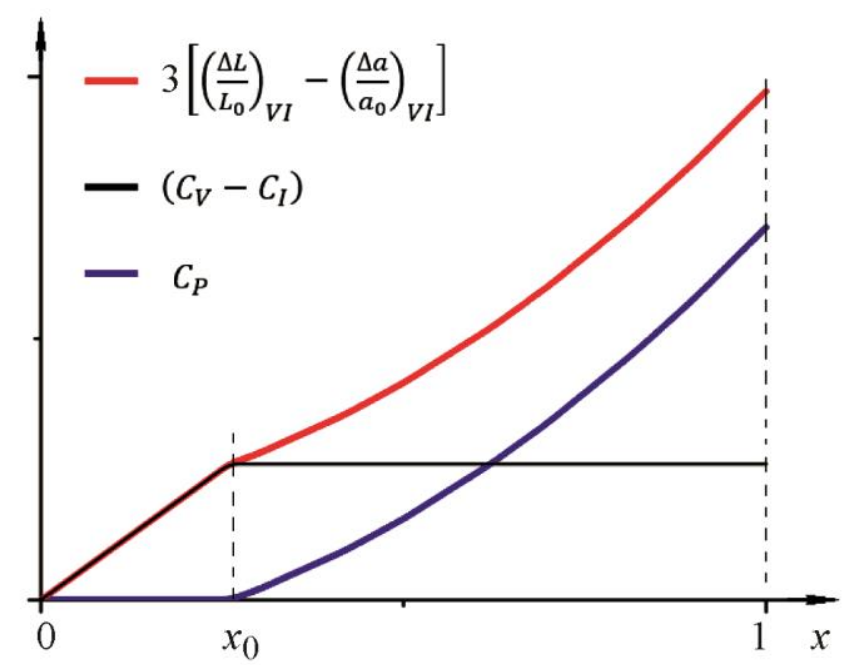

Рис. 3. Графическое выделение вклада вакансионных кластеров в распухание кристаллита

Отметим, что имеющиеся литературные данные по термическому разупорядочению, которое не способно создавать такие состояния, как нейтронное облучение, значительно более стойкого интерметаллического соединения $\mathrm{Ni}_{3} \mathrm{Al}$ показывают лишь простую линейную зависимость относительного приращения параметра решетки от параметра порядка [8].

Для контраста рассмотрим альтернативную модель, которая предполагает, что в каскадах происходит полное антиструктурное разупорядочение, а образующиеся же вакансии и междоузлия сохраняются в них же, причем в концентрациях, не зависящих от повторного перекрытия каскадов, а определяющихся лишь характеристиками материала.

Тогда, если вновь обозначить количество ячеек в каскадах как $N_{d}$, а концентрацию вакансий и междоузлий в них как $C_{V}^{(c a s)}$ и $C_{I}^{(c a s)}$ соответственно, то число вакансий и междоузлий во всем кристаллите будет равно:

$$
\left\{\begin{array}{l}
n_{V}=C_{V}^{(c a s)} 4 N_{d} \\
n_{I}=C_{I}^{(c a s)} 4 N_{d}
\end{array}\right.
$$

А их концентрации в масштабах всего кристалла:

$$
\left\{\begin{array}{l}
C_{V}=C_{V}^{(\text {cas })} C_{d}=C_{V}^{(\text {cas })}(1-S) \\
C_{I}=C_{I}^{(c a s)} C_{d}=C_{I}^{(c a s)}(1-S)
\end{array} .\right.
$$

Тогда их вклад в относительное приращение параметра решетки будет равен:

$$
\begin{gathered}
\left(\frac{\Delta a}{a_{00}}\right)_{V I}=\frac{1}{3}\left[C_{V}^{(\text {cas })} r_{V}+C_{I}^{(\text {cas })} r_{I}\right](1-S)=k_{V I} x \\
\left(\frac{\Delta a}{a_{00}}\right)_{\Sigma}=k_{A S} x+k_{V I} x=k_{\Sigma} x
\end{gathered}
$$


При этом никакого режима насыщения для вклада (47), а следовательно, и излома, аналогичного наблюдаемому на рис. 2, не предполагается. Отметим также, что поскольку в рамках данной модели выход междоузлий и вакансий за пределы каскада не предусматривался, то образующиеся дефекты являются только парами Френкеля, а поскольку никаких механизмов ухода дефектов на стоки не рассматривалось, то $C_{V}=C_{I}$, что, согласно (27), ведет к равенству относительного удлинения кристалла и относительного изменения параметра решетки.

\section{3. Заключение}

При рассмотрении наших моделей мы ограничивались простейшими дефектами Шоттки и Френкеля, а также вакансионными кластерами. Однако реальные системы радиационно-индуцированных дефектов, конечно, значительно сложнее. Например, хорошо известно [14], что стабильными конфигурациями для накопления междоузлий в ГЦК-системах являются не одиночные дефекты, а образованные из них «гантели» (double-bells). Но, как легко понять, это не препятствует использованию для их описания формул типа (16), где в таком случае под $r_{I}$ нужно понимать просто половину величины соответствующего коэффициента, определенного для такой пары. Конечно, помимо вакансионных кластеров существует масса и других протяженных дефектов - дислокаций, дислокационных петель и т. д., действующих как стоки точечных дефектов и тоже нуждающихся в учете.

На более глубоком уровне аналитическое описание радиационно-стимулированных процессов в материалах наиболее часто представляется в виде систем дифференциальных уравнений, описывающих баланс генерации, накопления и аннигиляции дефектов. Решением их и являются значения концентраций вакансий и междоузлий. Однако здесь следует еще раз подчеркнуть, что фактически дифракционные структурные исследования (рентгено- или нейтронографические), а также дилатометрия проводятся не «in situ», а после некоторого периода выдержки облученных образцов для уменьшения наведенной в них радиоактивности, т. е. некоего пострадиационного отжига. Соответственно оцениваемые с помощью приведенных здесь весьма простых формул концентрации дефектов относятся к их остаточным значениям. Тем не менее, можно надеяться, что даже столь простые модели могут оказаться полезными для качественного осмысления экспериментальных результатов и некоторых количественных оценок.

В частности, это уже оказалось полезным для анализа результатов радиационных эффектов в модельном соединении $\mathrm{Cu}_{3} \mathrm{Au}$. В дальнейшем мы планируем провести аналогичные работы с интерметаллидами $\mathrm{Ni}_{3} \mathrm{Ti}$ и $\mathrm{Ni}_{3} \mathrm{Al}$.

\section{Благодарность}

Работа выполнена с использованием УНУ «НМК ИФМ» в рамках государственного задания МИНОБРНАУКИ России (тема «Нейтрон» № АААА-А19-119112590082-1).

\section{Литература}

1. Neutron diffraction and electron microscopic investigation of decomposition and radiationinduced ageing of Cr-Ni-Ti austenitic alloys / V. M. Alab'yev, V. G. Vologin, S. F. Dubinin, S. S. Lapin, V. D. Parkhomenko, V. V. Sagaradze // Physics of Metals and Metallography. 1990. - Vol. 70, no. 2. - P. 131-137.

2. Precipitation hardening and radiation damageability of austenitic stainless steels / V. V. Sagaradze, V. M. Nalesnik, S. S. Lapin, V. M. Aliabev // Journal of Nuclear Materials. - 1993. - Vol. 202, no. 1-2. - P. 137-144. - DOI: 10.1016/0022-3115(93)90036-X.

3. Effects of titanium additions to austenitic ternary alloys on microstructural evolution and void swelling / T. Okita, W. G. Wolfer, F. A. Garner, N. Sekimura // Philosophical Magazine. 2005. - Vol. 85, no. 18. - P. 2033-2048. - DOI: 10.1080/14786430412331331871. 
4. Voronin V. I., Berger I. F., Goshchitskii B. N. Structural changes in a model alloy after irradiation of Fe62Ni35Ti3 with fast neutrons and isochronous temperature annealing // Physics of Metals and Metallography. - 2012. - Vol. 113, no. 9. - P. 878-882. - DOI: 10.1134/S0031918X12090141.

5. Neutron and X-ray diffraction analysis of the effect of irradiation dose and temperature on microstructure of irradiated HT-9 steel / P. L. Mosbrucker, D. W. Brown, O. Anderoglu, L. Balogh, S. A. Maloy, T. A. Sisneros, A. C. Dippel // Journal of Nuclear Materials. - 2013. Vol. 443, no. 1. - P. 522-530. - DOI: 10.1016/j.jnucmat.2013.07.065.

6. Peculiarities of radiation-induced processes in the Cr-Ni-Mo austenitic steels studied by neutron diffraction / V. I. Voronin, V. L. Arbuzov, V. I. Bobrovskii, S. E. Danilov, K. A. Kozlov, N. V. Proskurnina, V. V. Sagaradze // Diagnostics, Resource and Mechanics of materials and structures. - 2015. - Iss. 5. - P. 80-89. - DOI: 10.17804/2410-9908.2015.5.080-089.

7. Radiation induced disordering in $\mathrm{Cu} 3 \mathrm{Au} / \mathrm{N}$. V. Proskurnina, V. I. Bobrovskii, B. N. Goshchitskii, A. Yu. Volkov, V. I. Voronin // Radiation Physics and Chemistry. - 2020. Vol. 170. - P. 108654. - DOI: 10.1016/j.radphyschem.2019.108654.

8. Cahn R. W. Lattice parameter changes on disordering intermetallics // Intermetallics. 1999. - Vol. 7. - P. 1089-1094. - DOI: 10.1016/S0966-9795(99)00035-7.

9. Bhatia M. L., Cahn R. W. Lattice parameter and volume changes on disordering // Intermetallics. - 2005. - Vol. 13. - P. 474-483. - DOI: 10.1016/S0966-9795(99)00035-7.

10. Конобеевский С. Т. Действие облучения на материалы: введение в радиационное материаловедение. - М. : Атомиздат, 1967. - 402 с.

11. Kovács I., El Sayed H. Point defects in metals // J. Mater Sci. - 1976. - Vol. 11. P. 529-559. - DOI: 10.1007/BF00540934.

12. Beneagoub A., Thome L. Amorphization Mechanisms in Ion-Bombarded Metallic Alloys // Phys. Rev. B: Condens. Matter. - 1988. - Vol. 38. - P. 10205-10216. - DOI: 10.1103/PhysRevB.38.10205.

13. Eshelby J. D. The Continuum Theory of Lattice Defects // Solid State Physics. - 1956. Vol. 3. - P. 79-144. - DOI: 10.1016/S0081-1947(08)60132-0.

14. Was G. S. Fundamentals of Radiation Materials Science. Metals and Alloys. - 2nd ed. New York : Springer, 2017. - 1002p. - ISBN 978-1-4939-3436-2. - DOI: 10.1007/978-1-49393438-6. 